\title{
Criterios \\ El cd-rom Base de Datos de Intervenciones en edificios declarados Bien de Interés Cultural de Sevilla
}

Javier Lobato Domínguez

Archivo Histórico de FIDAS / COAS

\section{Resumen}

El cd-rom que ahora se difunde es fruto de la colaboración institucional entre la Fundación FIDAS, el IAPH y la Gerencia de Urbanismo del Ayuntamiento de Sevilla. El proyecto inicial consistió en la creación de una compleja base de datos cuyo objetivo era recopilar, describir y difundir toda la documentación relativa a acciones sobre el patrimonio inmueble monumental de Sevilla.

Tras varios años de trabajo incorporando en dicha base la información de los expedientes de intervención de los archivos de FIDAS, Gerencia de Urbanismo y del arquitecto Rafael Manzano, ha llegado el momento de dar a conocer los resultados aprovechando las posibilidades del medio y soporte informático.

\section{Palabras clave}

\section{CD-ROM}

Bases de datos

Bien de Interés Cultural

Fuentes de información

Patrimonio inmueble

Intervención

Sevilla

Instituto Andaluz del Patrimonio Histórico

Fundación FIDAS

Gerencia de Urbanismo del Ayto. de Sevilla
El cd-rom que ahora difundimos es fruto de una decidida y continuada colaboración institucional que se inició en 1997, entre la Fundación FIDAS y el IAPH, y a la que se incorporó en 2002 la Gerencia de Urbanismo del Ayuntamiento de Sevilla. Las tres instituciones tienen especial responsabilidad e interés por nuestro más valioso patrimonio inmueble, aquellos edificios hoy declarados Bien de Interés Cultural (BIC). Desde el Instituto Andaluz del Patrimonio Histórico se planteaba como necesario la correcta documentación de todos los inmuebles protegidos por ley, detaIlando su devenir histórico, características físicas y formales, autoría y datación; también se comprobó que era primordial conocer las intervenciones llevadas a cabo sobre los edificios, sus criterios, alcance y autoria ${ }^{1}$. Esta necesidad es también compartida por la Gerencia de Urbanismo del Ayuntamiento de Sevilla que durante las últimas décadas ha ido adquiriendo competencias patrimoniales, sobre todo tras la ampliación del Conjunto Histórico y el planeamiento de protección redactado para el mismo ${ }^{2}$, así como por los trabajos que lleva a cabo desde su Servicio de Conservación y Rehabilitación. Por último señalar la doble necesidad sentida por el colectivo de arquitectos, representado por su Colegio Profesional y Fundación FIDAS, de dar a conocer y poner en valor el rico patrimonio documental de su Archivo, y crear un moderno instrumento de documentación que facilitase el trabajo a los profesionales que se enfrentan a la muy dura y comprometida tarea de intervenir sobre un edificio histórico ${ }^{3}$.

Tras cinco años de intenso trabajo en los archivos de la Fundación FIDAS (fondos del Colegio de Arquitectos de Sevilla), en los diferentes fondos documentales de la Gerencia de Urbanismo del Ayuntamiento de Sevilla (archivo del servicio de Conservación, de Delineación, del antiguo departamento de Arquitectura, del servicio de Planeamiento, etc.), el archivo privado del arquitecto D. Rafael Manzano Martos, y la esencial contribución del Centro de Documentación del Instituto Andaluz del Patrimonio Histórico, se ha cubierto una etapa y conseguido importantes resultados. El principal ha sido la creación de una completa Base de Datos documental (BIC-Sevilla) en la que se reúne con carácter analítico, información de todas las intervenciones que hemos podido localizar (reformas, restauraciones, rehabilitaciones), e incluso de expedientes que aportan información sobre el inmueble (informes y certificados, estudios de patologías, levantamientos...) relativos a los 87 inmuebles declarados Bien de Interés Cultural de la ciudad de Sevilla ${ }^{4}$

Desde el primer momento nuestro trabajo se orientó hacia los preceptos de recopilación, custodia y difusión de toda la documentación relativa a acciones sobre el patrimonio monumental, que definían los manifiestos, reglamentaciones y programas internacionales, desde la famosa Carta de Atenas de 1931, pasando por la de Venecia de 1964 y por las precisas recomendaciones de las Cartas del Restauro de 1972 y 1987. En todos estos documentos se indica la necesidad de reunir en un archivo toda la documentación relativa a actuaciones sobre los monumentos y proceder a su difusión mediante publicaciones. Estas reco- 


\section{$076-077$ \\ Criterios}

El cd-rom Base de Datos de Intervenciones en edificios declarados Bien de Interés Cultural de Sevilla

\section{PH43 - Abril 2003}

mendaciones nunca se llegaron a cumplir de forma coherente y unitaria para el caso español, y sólo de manera parcial y limitada se realizaron esfuerzos en dicho sentido. El Ministerio de Cultura publicó en 1989 la obra "Fuentes documentales para el estudio de la restauración de monumentos en España"15 en la que se relacionaban los proyectos de intervención que custodiaba el Archivo del Ministerio. Para nuestro ámbito territorial y tras el nuevo marco legal que supuso la ley del Patrimonio Histórico Español y la transferencia de competencias a la Junta de Andalucía, se debe reseñar la difusión mediante publicaciones y exposiciones de algunas de las intervenciones más destacadas realizadas entre 1985 y $1989^{6}$. Por su parte la Gerencia de Urbanismo del Ayuntamiento de Sevilla también ha contribuido a la difusión de sus proyectos de intervención con sendas publicaciones relativas a la rehabilitación singular en el Conjunto Histórico de Sevilla (1996 y 1999) ${ }^{7}$. Frente a estos encomiables esfuerzos, el trabajo que aqui presentamos tiene dos cualidades que lo hacen único y, creemos, pionero en su género. Por un lado el aprovechamiento de los nuevos medios informáticos para recoger y estructurar la información documental, y por otro su afán totalizador (en palabras de François Houtart es necesario realizar un esfuerzo para mantener la utopía como aspiración).

Nuestra aportación se encamina por tanto hacia el conocimiento y difusión de experiencias de intervención bien documentadas, conservadas en nuestros archivos, expuestas de forma sistemática y objetiva, aprovechando en la mayor medida posible los medios y soportes informáticos. Todo ello con el propósito de crear un instrumento de información vivo, que se actualice y crezca con el tiempo.

A la fecha de hoy, la base de datos BIC-Sevilla contiene suficiente información (aún no toda la que desearíamos) ${ }^{8}$, relativa a expedientes, planos y fotografías como para constituir una fuente imprescindible para cualquier actuación en muchos de los inmuebles analizados, así como base de futuras investigaciones sobre los mismos. Llegados a este punto nos planteamos desde las tres instituciones que era absolutamente prioritario difundir el trabajo realizado, para hacer accesible este cuerpo documental a todos los profesionales e investigadores.

Cuando un arquitecto vaya a realizar en un futuro inmediato una obra de consolidación, restauración, o rehabilitación de alguno de estos inmuebles contará por tanto con planimetría actualizada del edificio, dispondrá de datos sobre la reciente historia material del inmueble, conociendo las áreas que fueron intervenidas en el pasado y con que criterios y materiales se realizaron. Por otro lado el historiador de la Arquitectura dispondrá de secuencias planimétricas fiables de un mismo edificio, o de varios que pertenezcan a una misma categoria: tipológica, estilística, etc. Sabrá con bastante precisión el alcance de las intervenciones sufridas y qué zonas o elementos se incorporaron durante las mismas. Tal vez evitemos así esa terrible sensación de inseguridad y

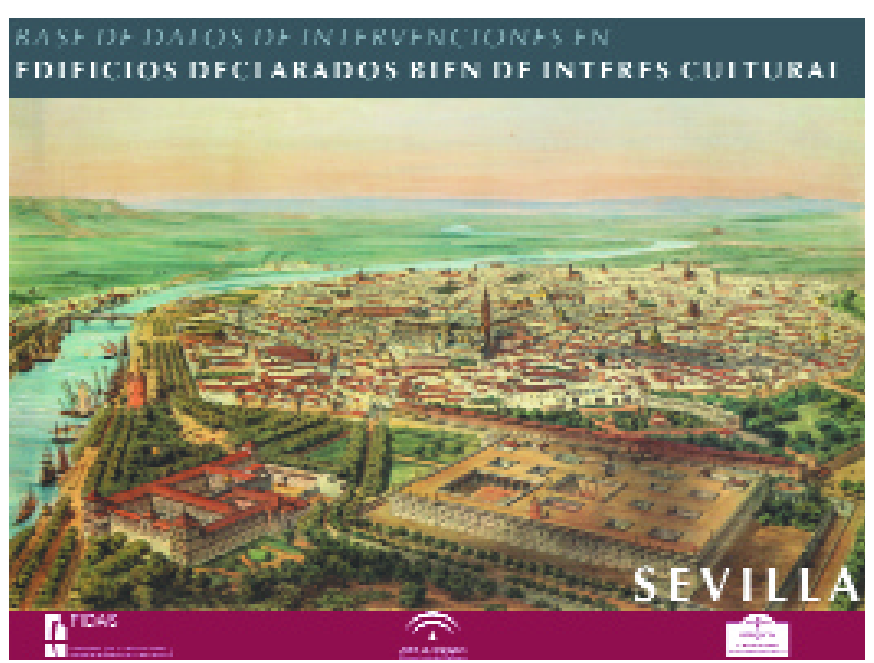

duda: "el temblor de la falsificación" utilizando las palabras de Patricia Highsmith, que en más de una ocasión nos asalta al analizar un edificio histórico.

Al editar el cd-rom, nos propusimos sin embargo ir más allá y aprovechar las posibilidades del medio (informático) y el mensaje contenido (Base de datos) para dotar de múltiples grados de lectura y capacidad de uso a la publicación. Por un lado los textos de introducción referentes a la ciudad, el plano interactivo de Sevilla -con la ubicación de los inmuebles--, los textos que analizan las distintas tipologías funcionales de los edificios, la precisa información histórica, descriptiva y bibliográfica aportada por el $\mathrm{IAPH}$, así como las propuestas de itinerarios y paseos para descubrir los inmuebles, dotan al cd-rom casi del carácter de una guía de arquitectura de nuestra ciudad, apta para todo tipo de público que desee acercarse al valioso patrimonio construido de Sevilla, todo ello sin perder el carácter instrumental antes aludido de fuente de trabajo y estudio para los profesionales. Por último un tercer aspecto es el constituido por las garantías que supone para la tutela de los inmuebles como testimonio de su integridad arquitectónica, y realidad física.

Queremos aclarar desde el primer momento que el cd-rom no es la base de datos BIC-Sevilla. Contiene información elaborada expresamente para el mismo (textos introductorios, módulo de Sevilla y tipologías, itinerarios, planimetría urbana de acceso), y por limitaciones técnicas y temporales no toda la información (planos y fotografías) de la base está contenida en el cd, en el que si constan todos los expedientes de intervención e informativos reseñados en la base hasta octubre de 2002 . Por otro lado señalaremos que integrar en un instrumento multimedia de difusión como es el cd la estructura y diferentes grados de información de una base de datos de gran complejidad ha sido tarea ardua; dificil para los programadores e informáticos y en ocasiones desesperante para quienes deseábamos que contuviese el máximo de información con la mayor claridad posible de utilización, todo ello con un diseño limpio y discreto que ayudase a la correcta legibilidad de los diferentes niveles de información?

Describiremos de forma muy breve cómo es el cd-rom para que lo anteriormente comentado se pueda comprender y valorar.

Hemos elegido como portada un grabado hasta hace pocos años no demasiado conocido y que con gran belleza y especial precisión congela la ciudad a vista de pájaro en 1860. La ciudad dibujada por Alfred Guesdon está aún casi intacta, confiada en la perennidad de sus muros... 


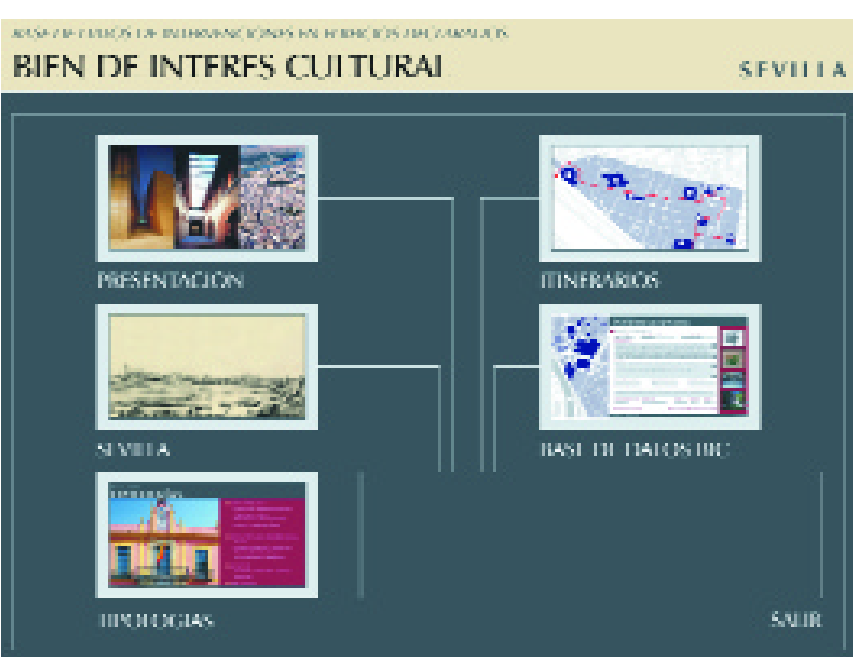

Tras las obligadas advertencias legales y técnicas se presenta una pantalla índice que sirve de mapa de navegación, en él encontramos los principales módulos que articulan el cd: Presentaciones; Sevilla ciudad y patrimonio edificado; Tipologías, Itinerarios y, cómo no, la propia Base de Datos.

Toda obra de esta entidad y complejidad requiere de presentaciones que indiquen qué propósitos e intenciones guiaron a sus autores a emprender y difundir su trabajo, en este caso contamos con las de las instituciones que lo han hecho posible, redactadas por sus responsables: Enrique Soler, Rafael Carmona y Román Fernández-Baca. Antecediéndolas una introducción global de Antonio Mena Anisi, secretario del COAS y del Patronato de FIDAS.

El primer módulo nos sitúa en la Ciudad. Desde el fenómeno urbano se estudia la incidencia de 2.800 años de historia sobre Sevilla. Un recorrido sobre las distintas ciudades que existieron y que con su velada presencia conforman y condicionan la urbe actual.

Debemos conocer también qué políticas de intervención y protección se aplican a este valioso legado arquitectónico. Los tres artículos que lo constituyen, redactados por experimentados arquitectos de la Gerencia de Urbanismo, nos plantean criterios e intenciones aplicados en las últimas décadas para que esta larga cadena de acciones continúe y permita mantener vivos nuestros más preciados inmuebles. Desde las más elementales prácticas encaminadas a la conservación, hasta las complejas operaciones de rehabilitación integral de algunos significativos edificios, todas estas intervenciones cuentan con las garantías técnicas y científicas de importantes equipos multidisciplinares.

Para terminar este apartado, se aporta un análisis cuantitativo de la base de datos. Se da así información cronológica del número de intervenciones, de su entidad (reformas, consolidaciones, restauraciones...), de qué instituciones y particulares las han promovido, y de quienes desde la especialización profesional han realizado mayor número de las mismas ${ }^{10}$. Los gráficos indican con precisión tendencias y periodos en la forma de tratar la intervención patrimonial: desde el predominio de reformas frente a restauraciones o rehabilitaciones en las décadas de los sesenta y setenta (fechas en la que las restauraciones aún se realizaban en sucesivas campañas anuales con proyectos y presupuestos independientes), hasta la preponderancia de importantes obras de rehabilitación durante los años ochenta y primera mitad de la década de los noventa.

Como aportación desde la docencia e investigación se incorporan varios estudios de especialistas en la materia, sobre las dife-

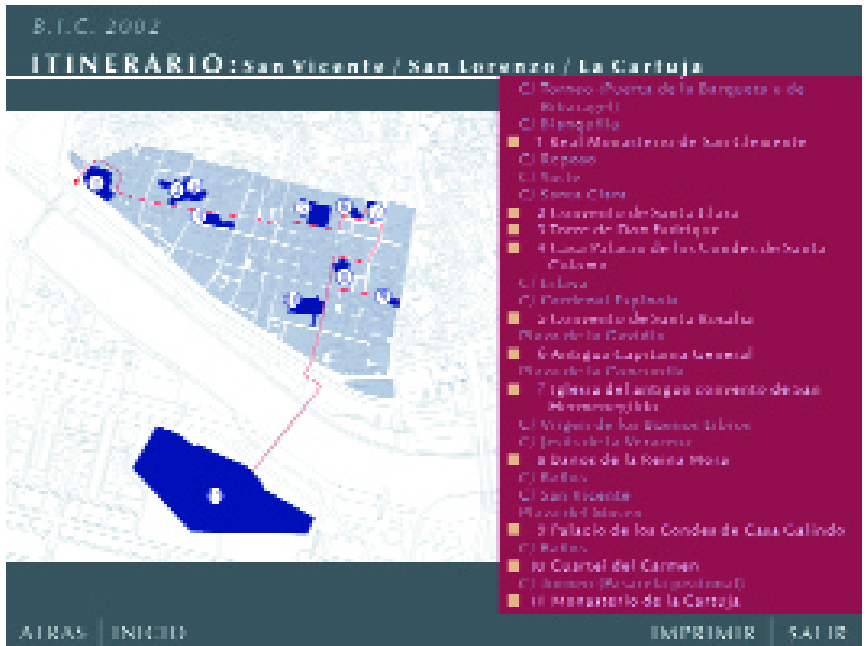

rentes tipologías en las que se encuadran los edificios declarados BIC: Arquitectura Civil Pública, Privada, Arquitectura Militar y Religiosa y sus diferentes subtipos. Es una magnifica oportunidad para pensar sobre nuestra arquitectura, su realidad espacial y funcional, asi como las peculiaridades que asumen en su implantación en la ciudad hispalense. Son arquitecturas que hacen ciudad y, a su vez están determinadas por el marco urbano en el que se insertan. ${ }^{11}$

Otro módulo es el constituido por los itinerarios, en el que a modo de propuesta se ofrecen varios paseos por la ciudad, para contemplar los diferentes edificios por su cercanía en un sector coherente de la urbe (recordemos cuantos edificios dotan de personalidad propia y singular a cada barrio) podemos así caminar por el conjunto monumental de los barrios de San Vicente y San Lorenzo, o por los principales inmuebles de interés de la judería.

El eje central del cd está constituido por la Base de Datos. Para poder acceder a los distintos inmuebles se parte de una secuencia de planos, vinculados a la lista de los edificios. Desde el ámbito de mayor amplitud geográfica: El municipio; hasta el Conjunto Histórico y su entorno inmediato, donde se ubican 83 de los edificios. Sobre este plano se realizan aproximaciones por distintos sectores, y en cualquiera de los mismos al marcar sobre el plano o sobre el nombre de la lista accedemos al registro del inmueble seleccionado.

La primera pantalla ofrece información general sobre el BIC: cronología, tipo, descripción física, datos históricos y legales. Todos ellos extractados de las bases de datos creadas por el $\mathrm{IAPH}$. Se aporta un grupo de fotografías significativas del inmueble, entre ellas siempre el plano de ubicación y una fotografía aérea; así como acceso a una importante selección bibliográfica (1.618 registros). Desde esta pantalla se puede también acceder a la lista de expedientes de intervención que han afectado al inmueble.

La lista de intervenciones ordenada cronológicamente nos muestra todas aquellas que están referenciadas en la base de datos, y que corresponden a las analizadas en los archivos de FIDAS, Gerencia de Urbanismo y de Rafael Manzano, asi como las incluidas desde la relación publicada por el Ministerio de Cultura en 1989. Los datos de fecha, titulo, autor y archivo permiten la rápida localización del expediente que interese consultar: marcando sobre él accedemos a los módulos documentales de la Base de Datos.

El primero describe de forma global el expediente, en esta pantalla se indican: signatura y archivo, año de inicio, tipo de inter- 


\section{$078-079$}

\section{Criterios}

El cd-rom Base de Datos de Intervenciones en edificios declarados Bien de Interés Cultural de Sevilla

\section{PH43 - Abril 2003}

vención, autores, título, promotor, descripción extractada de la memoria y observaciones. Desde ella podemos acceder a los documentos y planos que constituyen el expediente, o, en algunos casos, a las fotografías de la intervención.

Para profundizar aún más en la descripción debemos marcar sobre la línea Documentos / Planos que permite el acceso a la siguiente pantalla en la que se relacionan los documentos que forman el expediente (proyecto, o bien, proyecto básico, de ejecución, reformados, planos, informes, certificados, etc.) y se listan los planos que han sido seleccionados y de los cuales se dispone de imagen escaneada.

Si deseamos visualizar las imágenes debemos marcar sobre Acceso a planos, para dar paso a una nueva pantalla en la que figura la anterior lista de planos seleccionados y una ventana que muestra su imagen muy reducida. Los planos se pueden visualizar de forma rápida desde esta lista gracias a la imagen miniaturizada de los mismos que permite precisar cuales son los que interesa ver a mayor tamaño y, opcionalmente, proceder a su descarga ${ }^{12}$.

Somos nuestros primeros críticos, y sabemos que, a pesar del esfuerzo realizado, aún falta bastante para acercarnos a esa utopía totalizadora de la que hablamos antes. Es imprescindible para ello extender el trabajo a otros archivos ${ }^{13}$, así como mantener viva la base de datos mediante su constante actualización. También sería deseable ampliar el número de inmuebles, abarcando tanto los diferentes grados de protección, como extendiéndolo a otros ámbitos territoriales de nuestra comunidad. Continuidad y crecimiento se plantean como necesarios si queremos que la base de datos cumpla con los objetivos informativos, tutelares y patrimoniales señalados.

Por último se debe precisar que un trabajo de estas caracteristicas es fruto del trabajo colectivo de un cualificado grupo de profesionales de distintas disciplinas que han aportado su saber y también su entusiasmo ${ }^{14}$. A ellos se debe la valía y éxito de resultados del trabajo.

La siguiente tabla muestra de forma precisa los resultados del trabajo que publicamos en el cd-rom.
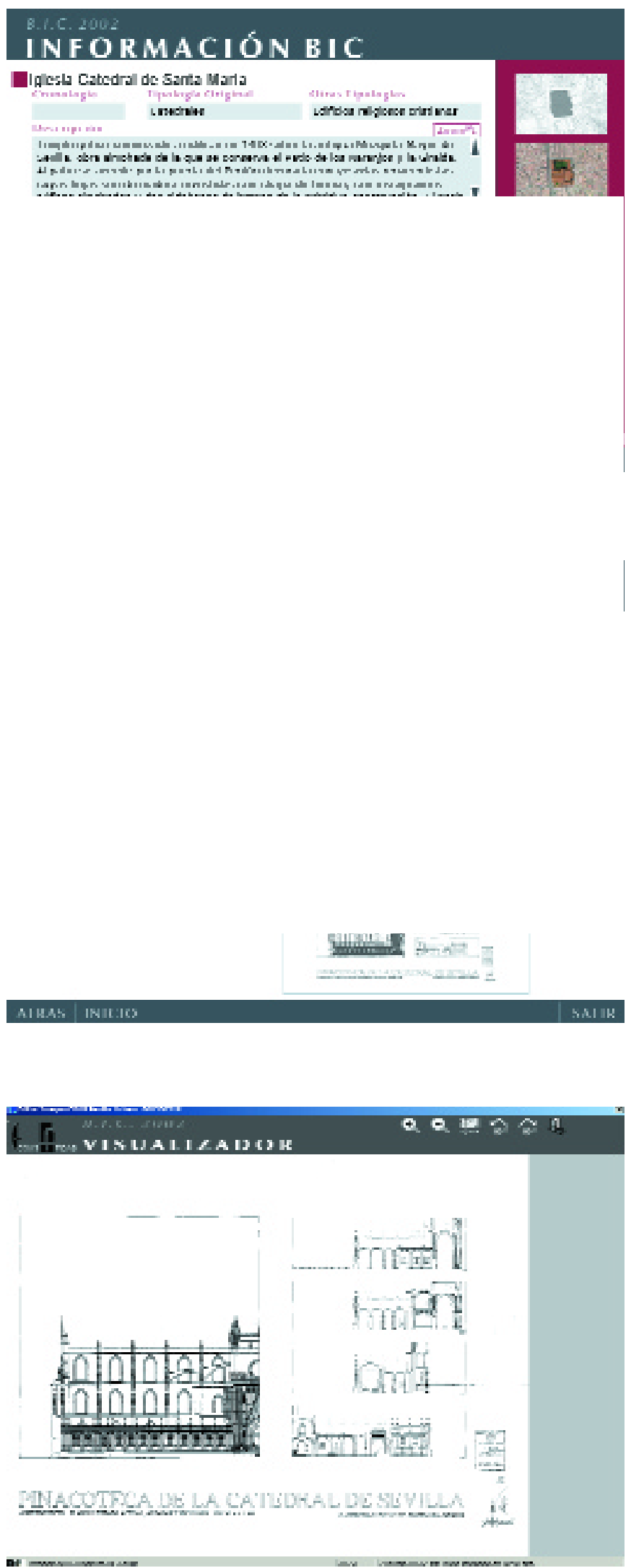


\section{Contenido del módulo base de datos del cd-rom BIC Sevilla}

$\begin{array}{lllllll}\text { Nombre BIC } & \text { Fotos BIC } & \begin{array}{l}\text { No } \\ \text { Intervenciones }\end{array} & \begin{array}{l}\text { Interv. Planos } \\ \text { MEC }\end{array} & \begin{array}{l}\text { Exp. } \\ \text { Informativos }\end{array} & \begin{array}{l}\text { Planos Exp. Informativos. } \\ \text { con fotos }\end{array} & \begin{array}{l}\text { Intervenciones } \\ \text { de Interv. }\end{array}\end{array}$

01 Antigua Audiencia Territorial

$3-0$

02 Antigua Capitanía General

03 Antigua Casa Consistorial

04 Antiguas Atarazanas Reales

05 Antiguo Almacén de Maderas del Rey

06 Antiguo Convento de la Merced

07 Antiguo Hospicio Prov. y Capilla Doméstica

08 Antiguos Palacio y Jardines de la Buhaira

09 Archivo General de Indias

10 Arco de la Plata

11 Baños de la Reina Mora

12 Capilla de la Encarnación del Señor

13 Capilla de San José

14 Capilla de $\mathrm{S}^{\underline{a}} \mathbf{M}^{\mathrm{a}}$ de Jesús del Antiguo Seminario 9

15 Cartuja de Santa María de Las Cuevas

16 Casa de Las Dueñas

17 Casa Museo de Murillo

18 Casa C/ Fabiola, 1

19 Casa de Las Columnas

20 Casa de los Pinelos

21 Casa de Olea

22 Casa de Pilatos

23 Casa del Rey Moro

24 Casa Palacio de los Condes de Sta. Coloma

25 Castillo de San Jorge

26 Colegiata del Divino Salvador

27 Coliseo España

28 Convento de la Santísima Trinidad

29 Convento de los Capuchinos

30 Convento de San Leandro

31 Convento de Santa Clara

32 Convento de Santa Inés

33 Convento de Santa María del Socorro

34 Convento de Santa Paula

35 Convento de $\mathrm{S}^{\mathrm{a}}$ Rosalía de Monjas Capuchinas

36 Convento e Iglesia de la Madre de Dios

37 Corral del Conde

38 Cuartel del Carmen

(Antiguo Convento de Carmelitas Calzados)

39 Edificio Asilo-Hospital de Ntra. Sa de la Paz

40 Edificio de la Antigua Univ. Literaria

41 Estación de la Plaza de Armas

42 Ex-Convento de los Remedios

43 Hospital de la Sangre

44 Hospital de San Lázaro

45 H. de Venerables Sacerdotes de S. Fernando

46 Iglesia Catedral de Santa María

47 Iglesia Convento de los Terceros y Restos del Palacio de los Ponce de León

4
4
4
4
2

1

2

1

17

48 Iglesia de la Magdalena y Capilla de la Hermandad de Nuestra Señora de la Antigua

49 Iglesia de Omnium Sanctorum 
$080-081$

\section{Criterios}

El cd-rom Base de Datos de Intervenciones en edificios declarados Bien de Interés Cultural de Sevilla

\section{PH43 - Abril 2003}

\begin{tabular}{|c|c|c|c|c|c|c|c|c|c|c|}
\hline & Nombre BIC & Fotos BIC & $\begin{array}{l}\text { № } \\
\text { Intervenciones }\end{array}$ & $\begin{array}{l}\text { Interv. } \\
\text { MEC }\end{array}$ & Planos & $\begin{array}{l}\text { Exp. } \\
\text { Informativos }\end{array}$ & $\begin{array}{l}\text { Planos Exp. } \\
\text { Informativos. }\end{array}$ & $\begin{array}{l}\text { Intervenciones } \\
\text { con fotos }\end{array}$ & $\begin{array}{l}\text { Fotos } \\
\text { de Interv. }\end{array}$ & Bibliografia \\
\hline 50 & Iglesia de San Benito de la Calzada & 3 & 6 & 0 & 3 & 0 & 0 & 0 & 0 & 6 \\
\hline 51 & Iglesia de San Esteban & 4 & 4 & 3 & 17 & 0 & 0 & 0 & 0 & 12 \\
\hline 52 & Iglesia de San Gil & 3 & 1 & 0 & 0 & 0 & 0 & 0 & 0 & 16 \\
\hline 53 & Iglesia de San Luis & 7 & 2 & 0 & 14 & 1 & 0 & 0 & 0 & 29 \\
\hline 54 & Iglesia de San Marcos & 6 & 16 & 13 & 4 & 2 & 3 & 0 & 0 & 22 \\
\hline 55 & Iglesia de Santa Ana de Triana & 5 & 13 & 4 & 32 & 1 & 4 & 1 & 2 & 31 \\
\hline 56 & Iglesia de Santa Catalina & 6 & 8 & 3 & 13 & 1 & 2 & 0 & 0 & 28 \\
\hline 57 & Iglesia de Santa María de las Nieves & 7 & 10 & 3 & 12 & 0 & 0 & 3 & 27 & 17 \\
\hline 58 & Iglesia de Santa Marina & 4 & 17 & 11 & 12 & 0 & 0 & 1 & 3 & 21 \\
\hline 59 & I. del Antiguo Convento de S. Hermenegildo & 3 & 18 & 9 & 54 & 0 & 0 & 0 & 0 & 18 \\
\hline 60 & Iglesia Parroquial de San Isidoro & 4 & 5 & 1 & 10 & 0 & 0 & 0 & 0 & 24 \\
\hline 61 & Iglesia Parroquial de San Jacinto & 4 & 6 & 0 & 33 & 0 & 0 & 0 & 0 & 3 \\
\hline 63 & Museo Arqueológico & 5 & 6 & 0 & 14 & 0 & 0 & 0 & 0 & 80 \\
\hline 64 & Palacio Arzobispal & 5 & 19 & 6 & 32 & 0 & 0 & 0 & 0 & 17 \\
\hline 65 & Palacio de Altamira & 4 & 8 & 2 & 27 & 0 & 0 & 1 & 1 & 10 \\
\hline 66 & Palacio de la Condesa de Lebrija & 4 & 5 & 0 & 1 & 0 & 0 & 0 & 0 & 4 \\
\hline 67 & Palacio de los Condes de Casa-Galindo & 4 & 6 & 0 & 26 & 0 & 0 & 0 & 0 & 3 \\
\hline 68 & Palacio de Miguel de Mañara & 5 & 10 & 1 & 34 & 2 & 0 & 0 & 0 & 13 \\
\hline 69 & Palacio de San Telmo y Jardín contiguo & 3 & 7 & 2 & 10 & 0 & 0 & 0 & 0 & 46 \\
\hline 70 & Patio del Antiguo Convento de San Acasio & 4 & 9 & 0 & 13 & 0 & 0 & 0 & 0 & 2 \\
\hline 71 & Plaza de Toros de la Real Maestranza & 4 & 59 & 0 & 26 & 3 & 2 & 1 & 1 & 21 \\
\hline 72 & Posada del Lucero & 6 & 2 & 0 & 2 & 1 & 6 & 0 & 0 & 3 \\
\hline 73 & Postigo del Aceite & 3 & 1 & 0 & 2 & 0 & 0 & 0 & 0 & 1 \\
\hline 74 & Puente de Isabel II & 5 & 5 & 1 & 6 & 1 & 1 & 0 & 0 & 7 \\
\hline 75 & Real Casa de la Moneda & 6 & 35 & 0 & 153 & 2 & 20 & 3 & 10 & 20 \\
\hline 78 & Murallas de la Macarena & 4 & 18 & 10 & 83 & 0 & 0 & 3 & 17 & 30 \\
\hline 79 & Reales Alcázares & 6 & 22 & 15 & 22 & 2 & 8 & 2 & 48 & 181 \\
\hline 80 & Restos del antiguo Convento de S. Agustín & 2 & 3 & 0 & 28 & 5 & 42 & 2 & 27 & 13 \\
\hline 81 & Templete de la Cruz del Campo & 3 & 1 & 0 & 0 & 1 & 1 & 0 & 0 & 12 \\
\hline 82 & Torre de Abdelazis & 3 & 1 & 0 & 6 & 0 & 0 & 0 & 0 & 5 \\
\hline \multirow[t]{2}{*}{83} & Templete y Monasterio de San Jerónimo & & & & & & & & & \\
\hline & de Buenavista & 5 & 13 & 2 & 90 & 9 & 33 & 2 & 13 & 12 \\
\hline 84 & Torre de Don Fadrique & 3 & 3 & 0 & 24 & 2 & 1 & 0 & 0 & 12 \\
\hline 85 & Torre de la Hacienda de Miraflores & 4 & 5 & 0 & 38 & 1 & 11 & 1 & 6 & 2 \\
\hline 86 & Torre de la Plata & 6 & 4 & 0 & 44 & 1 & 0 & 2 & 13 & 3 \\
\hline \multirow[t]{2}{*}{87} & Torre del Oro & 4 & 6 & 2 & 10 & 1 & 6 & 1 & 12 & 48 \\
\hline & & 419 & 769 & 195 & 2229 & 70 & 184 & 44 & 281 & 1618 \\
\hline
\end{tabular}

Inmuebles: 87

Expedientes referenciados: $(769+70)=839$

Expedientes consultados y descritos: $(574+70)=644$

Total fotos: $(419+281)=700$

Total planos: $(2229+184)=2413$

Registros bibliográficos:1618 
${ }^{1}$ Los instrumentos documentales creados para tal fin por el Centro de Documentación del IAPH son bien conocidos y han sido objeto de varios artículos en esta misma publicación entre ellos destacamos los siguientes:

LADRÓN DE GUEVARA SÁNCHEZ, Carmen. El Centro de Documentación del Patrimonio Histórico: Objetivos, gestión, servicios. En PH Boletín del Instituto Andaluz del Patrimonio Histórico. $n^{0} 15$ (junio 1996) p. 104 y ss.

PICO VALIMAÑA, Ramón; PÉREZ HUMANES, Mariano A. Patrimonio arquitectónico en el Sistema de Información del Patrimonio Histórico Andaluz. En PH Boletín del Instituto Andaluz del Patrimonio Histórico. $n^{\circ} 17$ (diciembre 1996) p.100 y ss

PÉREZ CANO, Ma del Valle; PÉREZ HUMANES, Mariano; TAPIA MARTín, [et al.] Desarrollo de la base de datos inmuebles: Algunas reflexiones sobre el destino de la documentación patrimonial. En PH Boletín del Instituto Andaluz del Patrimonio Histórico. $n^{\circ} 19$ (junio 1997) p. 131 y ss.

PICO VALIMAÑA, Ramón; PÉREZ HUMANES, Mariano A. Patrimonio arquitectónico y urbanistico en Andalucia: hacia la configuración de un sistema de información territorial. En PH Boletín del Instituto Andaluz del Patrimonio Histórico. nº 22 (marzo 1998) p. 106 y ss.

JUAN Y SANTOS, Luisa Fernanda de. El proyecto de fuentes documentales del patrimonio histórico y su informatización. En PH Boletín del Instituto Andaluz del Patrimonio Histórico. n²8 (septiembre 1999) p.153 y ss.

MUÑOZ CRUZ, Valle; ORTEGA VAQUERO, Isabel. La base de datos del Patrimonio Inmueble de Andalucía en Internet: BDI-PHA.. En PH Boletín del Instituto Andaluz del Patrimonio Histórico. $n^{\circ} 36$ (septiembre 2001) p. 238 y ss.

MUÑOZ CRUZ, Valle. Hacia un Sistema Integrado de Patrimonio Histórico de Andalucia: la Base de Datos del Patrimonio Inmueble. En PH Boletín del Instituto Andaluz del Patrimonio Histórico. n³7 (diciembre 2001) p.223 y ss.

${ }^{2}$ Sobre las competencias municipales en materia de patrimonio y el planeamiento de protección aprobado ver El Conjunto Histórico de Sevilla Avance del Plan Especial de Protección / Ayuntamiento de Sevilla. Gerencia de Urbanismo ; José Garcia Tapial...[et al.]. - Sevilla: Ayuntamiento. Gerencia de Urbanismo, 1995. El estado de aprobación de los diferentes planes parciales en octubre de 2002 se analiza en el artículo de Juan Garcia Gil: Desarrollo de Plan Especial de Protección del Conjunto Declarado, texto que se incluye en el cd-rom que aquí presentamos.

${ }^{3}$ Ya explicamos los objetivos, alcance y posibilidades de la base de datos BIC-Sevilla, as como el interés de la institución colegial por estos temas en nuestro artículo: Intervención en el Patrimonio Inmueble: valor heurístico e instrumental de la base de datos BIC-Sevilla. En FIDAS: Publicación Periodica. Nº 0 (enero 2002) p.22-27.

${ }^{4}$ Sobre la estructura inicial de la base y el proceso de documentación seguido ver: LOBA TO DOMINGUEZ, Javier ; FRAUCA BELLOSO, Carmen; ACEME DAVILA, Antonio: Informatización de la planimetría de los inmuebles BIC de Sevilla incluidos en el archivo histórico de la Fundación FIDAS. En PH Boletín del Instituto Andaluz del Patrimonio Histórico. n²6 (abril 1999) p.157 y ss. Si bien desde entonces la base se ha modificado para permitir incluir la información aportada por el IAPH y la Gerencia de Urbanismo del Ayuntamiento de Sevilla.

${ }^{5}$ Fuentes documentales para el estudio de la restauración de monumentos en España / Prólogo de Alfonso Muñoz Cosme ; introducción Soledad Cases Gómez de Olmedo . - Madrid: Ministerio de Cultura, 1989. La publicación referencia 8.556 proyectos de intervención realizados en toda España durante el periodo comprendido entre el final de la guerra civil y los años ochenta, cuando se produce las transferencias de competencias a las diferentes comunidades autónomas (Andalucia en 1984). Aún así es significativa la observación de Soledad Cases cuando dice "La multiplicidad de órganos administrativos que han intervenido respecto a bienes del patrimonio artístico a partir de la guerra civil ha originado una dispersión de los fondos documentales correspondientes."

${ }^{6}$ Destacamos la publicación del libro Conservación y Restauración de Bienes Culturales en Andalucía: Primeras experiencias / Alicia Martinez Martín, Lucas Manuel Espinosa de los Monteros Choza. - Sevilla: Junta de Andalucia, Consejería de Cultura, Dirección General de Bienes Culturales, 1999. Obra imprescindible para conocer las políticas y métodos de intervención en las primeras etapas autonómicas, si bien, la voluntad, manifestada en la introducción de esta publicación, de darle continuidad editando nuevos volúmenes y finalmente un anuario que recogiese de forma sistemática todas las intervenciones no se ha cumplido aún.

${ }^{7}$ El Conjunto Histórico de Sevilla: Rehabilitación Singular / José Nuñez Castaín ; Redacción Reyes Morales Hevia, Ana Álvarez Marcos. - Sevilla: Ayuntamiento. Gerencia de Urbanismo, 1996. Y El Conjunto Histórico de Sevilla: Rehabilitación Singular II / Redacción Servicio de Paisaje Urbano y Patrimonio. - Sevilla: Ayuntamiento. Gerencia de Urbanismo, 1999. En las dos publicaciones se extractan brevemente las intervenciones promovidas por la Gerencia de Urbanismo, cada descripción se acompaña de valiosas fotografias de estado inicial y final, hecho que contrasta con la nula presencia de planimetria. También llama la atención que no figuren los nombres de los arquitectos y técnicos responsables de los proyectos.

${ }^{8}$ A pesar de los más de 800 expedientes y 2700 planos referenciados, somos plenamente conscientes de las carencias de la base y el cd. Los fondos de archivo estudiados contie- nen gran número de expedientes relativos a proyectos de intervención, pero no todos los que se han realizado. Aún limitándonos a los últimos 50 años (el Colegio de Arquitectos se fundó en 1931 pero la obligación de dejar copia de los trabajos visados sólo se remonta a 1949, mientras que la Gerencia de Urbanismo como organismo autónomo se crea en los años 80 ), nos consta que hay proyectos que no se han visado, ni han dejado testimonio documental en la Gerencia Municipal. Entre otros es el caso de los proyectos realizados por la Diputación Provincial en el Hospital de San Lázaro, o la Iglesia de San Luis, y también de las realizadas por otros organismos municipales en el Mercado de Triana (Castillo de San Jorge). Esperamos poder resolver estas ausencias en el futuro ampliando el trabajo a otros Archivos.

${ }^{9}$ En este sentido hay que señalar que el visualizador de planos que utilizamos en el cd-rom es fruto del esfuerzo y capacidad de los informáticos del COAS: Antonio Acemé Dávila, José María Fernández, y José Palomino. Gran parte del diseño general y maquetación de los planos urbanos se debe al futuro arquitecto Rafael Borondo García.

${ }^{10}$ Articulos FIDAS: publicación periódica $n^{\circ} 3$ (2002).

${ }^{11}$ Son un total de 14 artículos realizados por arquitectos, historiadores y un arqueólogo, según la propuesta de división tipológica que planteamos:

- Arquitectura Civil Pública: Edificios Administrativos / Mercedes Linares Gómez del Pulgar. - Arquitectura Civil Pública: Fábricas Reales / Mercedes Espiau Eizaguirre. Arquitectura Civil Pública: Territorio - Transportes / Rafael Borondo Garcia - Arquitectura Civil Pública: Ocio y Cultura / Lidia Suárez Samaniego

Arquitectura Civil Privada Residencial: Casas / Álvaro Jiménez Sancho

Arquitectura Civil Privada Residencial: Casas - Palacios y Palacios / Francisco Ollero Lobato Arquitectura Civil Privada Residencial: Vivienda Colectiva - Alojamiento Temporal / Mercedes Espiau Eizaguirre.

- Arquitectura Militar: Castillo, Torres, Muralla / Alfonso Jiménez Martín

- Arquitectura Religiosa: Iglesias y Capillas: Parroquias / Pedro Gómez de Terreros Guardiola Arquitectura Religiosa: Iglesias y Capillas: Capillas / Mercedes Pérez del Prado Arquitectura Religiosa: Iglesias y Capillas: Catedral / Javier Lobato Domínguez - Arquitectura Religiosa: Conventos y Monasterios / Ma Teresa Pérez Cano, Eduardo Mosquera Adell Arquitectura Religiosa: Hospitales / Francisco Pinto Puerto

- Arquitectura Religiosa: Humilladeros / Manuela Pérez Romero

12 Hay 2413 planos en el cd, 1281 de ellos se ofrecen en tamaño y resolución original con la posibilidad de ser descargados a disco. El resto se encuentran en formato reducido.

${ }^{13}$ Ya comentábamos la multiplicidad de archivos y fondos documentales que conservan expedientes de intervención en inmuebles de interés patrimonial. Soledad Cases lo indicaba para el ámbito estatal y desde el propio IAPH se está acometiendo el trabajo de realizar una guia informatizada de fuentes documentales del Patrimonio (vid JUAN Y SANTOS, Luisa Fernanda de: El proyecto de fuentes documentales del patrimonio histórico y su informatización. Op. Cit.). Para seguir avanzando en nuestro proyecto se debería ampliar el proceso documental a los archivos de rango autonómico (Central de la Consejería de Cultura, de la Delegación Provincial de Sevilla y de la Dirección General de Bienes Culturales), por otro lado estamos avanzando en la catalogación informatizada del Archivo del arquitecto Rafael Manzano Martos, quien fue arquitecto de zona del Ministerio para Andalucía Occidental y Badajoz desde 1963 hasta los años setenta. Este sistema de arquitectos de zona estuvo en vigencia desde los años veinte del siglo pasado y supuso la acaparación por parte de sus titulares de los más importantes proyectos de restauración sobre el patrimonio monumental. Alfonso Jiménez (Manca Finezza nel Restauro. En Un siglo de Arquitectura a través del Archivo de FIDAS / COAS. - Sevilla: FIDAS, 2002) indica quienes fueron los cinco arquitectos que ocuparon dicho cargo en nuestro ámbito territorial: Pablo Gutiérrez Moreno (1923?1930), José Maria Rodríguez Cano (1930-1936), Antonio Gómez Millán (1936-1938), Félix Hernández Giménez (1939-1968?) y Rafael Manzano Martos (1963?-1968?). El pasado año fue cedido a FIDAS el archivo profesional de Antonio Gómez Millán, con lo que dispondremos documentación de dos de los arquitectos citados. El archivo de Félix Hernández se conserva en el Museo Arqueológico de Córdoba y sería de gran interés para nuestra base de datos poder acceder a sus proyectos. Finalmente indicar que los fondos municipales anteriores a 1969 se encuentran en el Archivo Histórico Municipal de Sevilla, un fondo de gran riqueza aún poco explorado.

Debemos finalmente precisar que lo que describimos en la base de datos BIC-Sevilla son expedientes de archivo y en ocasiones repetimos una misma intervención en dos archivos diferentes, nuestra experiencia nos indica que rara vez el expediente de una misma intervención es idéntico en los dos archivos, esto es sobre todo notorio para los conservados en los archivos profesionales de los arquitectos frente a los presentados en las diferentes administraciones.

${ }^{14}$ Además de las personas que han trabajado directamente en la base de datos y el cd y que aparecen citados en los créditos, agradecemos muy especialmente la ayuda de todos aquellos que desde las instituciones y archivos, tanto en el IAPH, Gerencia de Urbanismo y Archivo Municipal, han facilitado nuestra tarea. Es por otro lado obligado reseñar que la mayor parte del esfuerzo de documentación ha recaído en tan sólo dos personas: Carmen Moliz Bonilla y Carmen Frauca Belloso. 